\title{
Performance Improvement of Routing Protocol in Ad-hoc Networks Using Smart Antenna with Balance Load
}

\author{
Arvind Kumar and Rajeev Tripathi, Member, IEEE
}

\begin{abstract}
We have presented a balanced routing protocol for Mobile Ad-hoc Networks using the smart antenna in this paper. Multipath routing can be effectively used to maximize the utilization of network resources for real-time traffic. In mobile ad-hoc network, this approach can suffer from co-channel interference or route coupling due to simultaneous transmission of packets via multiple routes. We use zone-disjoint routes and define maximally zone disjointness as route selection criteria. An adaptive routing strategy is proposed that use the advantages of directional antennas in ad hoc networks through the selection of maximally zone disjoint routes. Zone-disjoint routes would reduce the route coupling effect in wireless media by selecting routes in such that it produce minimal interference among communication over multiple routes. The proposed routing protocol provide effective load balancing in wireless media and it is implemented for both single path and multipath routing on ad hoc networks with directional antennas. Simulation results show that zone-disjoint single path routing can produce better throughput compare to multipath and AODV (Ad-hoc on-demand distance vector) routing protocol for more number of simultaneous communications.
\end{abstract}

Index Terms-Ad-hoc network, Directional antenna, Multipath routing, Zone-disjointness,

\section{INTRODUCTION}

Wireless technology is one of the hottest topics in computing and communication. Wireless network technology offer high data rate for mobile users, which enables the handling of multimedia Web content, videoconferencing, e-commerce, etc. Routing is one of the key issues for supporting these demanding applications in unstable and resource limited wireless networking environment. Mobile ad-hoc networks (MANETs) are collection of wireless nodes, dynamically connected without any infrastructure. Due to flexibility it may be used as communication network in rescue operation in emergency caused by the disasters, and battle field. Other potential applications of ad-hoc networks are sensor networks, community networking, home and office indoor networking etc.

MANETs are infrastructure-less mobile networks, each node communicates with other nodes directly or indirectly through intermediate nodes. Thus, all nodes are virtually routers participating in routing and routing protocols are required for selecting the routes and storing the information of routes. MANETs pose substantially different challenges to networking protocol than traditional wired networks. An enamors number of routing protocol have been developed for MANETs [1-8], which are characterized by dynamic network topology changes, quality of service, high degree of mobility, energy-constrained, bandwidth-constrained, intermittent connection, application oriented, and memory constrained, etc. The routing problem has not been well researched in infrastructure-less wireless networks, where goals are efficient route detection and adaptation to the network topology, scalability, and convergence. It is difficult to find the unique solutions since MANETs are inherently more dynamic. The key techniques that can be used to overcome these problems are smart antennas and modified MAC (Medium access control), and routing protocols.

Directional antenna is a very efficient and low cast smart antenna technology that application in ad-hoc networks had been studied over the last few years. Directional antenna can achieve better performance, higher throughput, and better resource utilization. Impact of directional antenna on the performance of DSR (Dynamic source routing) protocol has been investigated and proposed some modifications in original DSR designed for omni-directional antenna, leading to directional DSR (DDSR) [9]. In this paper authors had provided a trade off involve in using directional antenna in ad-hoc networks. Multipath routing protocols and routing protocols using the directional antenna for ad-hoc networks has been investigated [10-14]. Authors had analyzed the problem of route coupling with omni-directional antenna and proposed a mechanism to alleviate the problem using directional antenna. Routing performance using multiple paths improves substantially with directional antenna compared to that with omni-directional antenna [10]. A multipath routing in directional antenna ad hoc networks (MDAR) is proposed in [11]. In this paper several issues related to effective routing in directional antenna ad hoc networks are addressed and modifications to multipath routing for directional antennas are introduced. An on-demand Split Multipath Routing (SMR) protocol for ad-hoc network has been presented [12] that utilize multiple routes of maximally disjoint paths. Two directional routing protocols have been proposed [13], in order to enhance the performance of ad-hoc networks using directional antennas. Theses directional routing protocols avoid interference from nodes hops away by exploiting the directionality of the beams. In [14], authors have proposed a DDSR (directional-DSR) based multipath protocol to enhance the routing performance of ad-hoc networks using directional antenna. 
For best utilization of directional antennas, a suitable MAC protocol must be designed. Modification required in current MAC protocols and new MAC protocols for ad-hoc networks using directional antenna has been presented in [15-17]. Although smart antennas are a physical-layer technique and ad-hoc networking is a medium access control (MAC) layer technique, and routing protocol is network layer techniques, one should not assume that the two techniques can be implemented independently.

This paper is organized as follows: In Section II a brief introduction of zone-disjoint routes using omni-directional and directional antenna is given. Section III describes the system model that includes the basic concepts and definitions of directional antenna, network information, multipath routing using omni-directional antenna and directional antenna. Performance evaluation is given in Sections IV that discusses the simulation setup and performance under the static and dynamic environments. Finally we conclude with future scope of works in Section V.

\section{ZONE-DISJOINTNESS}

Let us consider the scenario given in Fig.1, where two source nodes $T_{1}$ and $T_{2}$ are communicating with receivers $R_{1}$ and $R_{2}$. Source $T_{1}$ to destination $R_{1}$ communicate through intermediate nodes $\mathrm{N}_{1}$ and $\mathrm{N}_{2}$. At the same time, $\mathrm{T}_{2}$ also wants to communicate with $\mathrm{R}_{2}$ through three possible routes $\left\{\mathrm{T}_{2}, \mathrm{~N}_{1}, \mathrm{~N}_{2}, \mathrm{R}_{2}\right\},\left\{\mathrm{T}_{2}, \mathrm{~N}_{3}, \mathrm{~N}_{4}, \mathrm{R}_{2}\right\}$ and $\left\{\mathrm{T}_{2}, \mathrm{~N}_{5}, \mathrm{~N}_{6}, \mathrm{R}_{2}\right\}$. If $\mathrm{T}_{2}$ selects the first or second route, routing performance will be deteriorate due to route coupling. Hence only directional antenna cannot improve the routing performance due to path overlap and route coupling of the nodes.

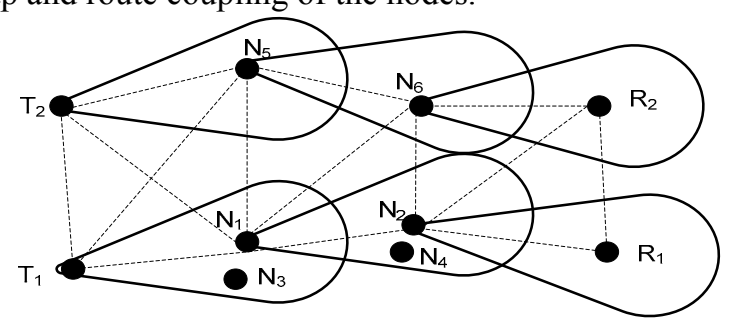

Fig.1: Zone-disjoint communication with directional antenna

Route coupling occurs when two routes are located physically close enough to interfere with each other during communication. As a result, the nodes in those two routes are constantly contending for access to the medium they share. Nodes belong to these two routes are within the transmission zone of one another, these two communications cannot happen simultaneously. $\mathrm{N}_{1}$ and $\mathrm{N}_{3}$ cannot receive data simultaneously from $T_{1}$ and $T_{2}$ respectively. Similarly, $N_{2}$ and $\mathrm{N}_{4}$ cannot receive data simultaneously from $\mathrm{N}_{1}$ and $\mathrm{N}_{3}$, respectively.

The two routes $\left\{\mathrm{T}_{1}, \mathrm{~N}_{1}, \mathrm{~N}_{2}, \mathrm{R}_{1}\right\}$ and $\left\{\mathrm{T}_{2}, \mathrm{~N}_{5}, \mathrm{~N}_{6}, \mathrm{R}_{2}\right\}$ are coupled with each other, by using omni-directional antennas as shown with dotted lines in Fig 1. But they are completely decoupled, if we use directional antennas. These two routes are said to be zone-disjoint, because data communication over one path will not interfere with data communication over the other path. Hence zone-disjoint routes ensure effective load balancing in the network. Zone-disjointness alone is also not sufficient for performance improvement.
Path length is also another important factor. A longer path with a larger number of hops will increase the end to-end delay and wastage of network bandwidth, even in the context of zone-disjointness. So, it will be better to select maximally zone-disjoint shortest paths. Zone-disjoint shortest path routing scheme being used in multipath routing and show the performance improvement of multipath routing over single-path routing under low traffic load.

\section{SYSTEM MODEL}

\section{A. Basic concepts}

When a node $\mathrm{n}$ forms a transmission beam at an angle $\theta$ and a beam width $\Phi$ with a transmission range $\mathrm{R}$, the coverage range at an angle $\theta$ is defined as transmission_zone ${ }_{n}$ $(\theta, \Phi, \mathrm{R})$ of node $\mathrm{n}$ as shown in Fig. 2 . If a node $\mathrm{n}$ is with the transmission_zone $\mathrm{n}_{\mathrm{n}}(\theta, \Phi, \mathrm{R})$ and other node $\mathrm{m}$ is in receive mode, then whenever $\mathrm{n}$ transmits a message at that transmission angle $\theta$ with respect to $\mathrm{n}$, beam width $\Phi$ and transmission range $\mathrm{R}$, it will be received by $\mathrm{m}$. When node $\mathrm{m}$ moves out of the transmission_zone $e_{n}(\theta, \Phi, R)$ the connectivity between $\mathrm{n}$ and $\mathrm{m}$ will be broke.

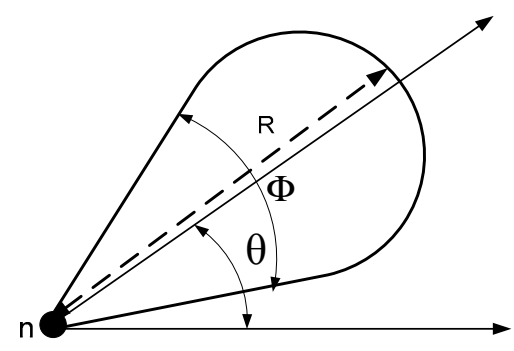

Fig.2: Transmission Zone of node $\mathrm{n}(\theta, \Phi, \mathrm{R})$

Route coupling occurs when two routes are located physically close enough to interface with each other during data communication. Transmission zone used by one communication interfere with the transmission zone used by other communication. Contention between two communications to access the wireless medium will result in high end-to end delay and performance of each interfering transmission will suffer. Network performance can be improved by reducing the coupling between two interfering routes. Correlation factor $\eta$ is used to measure route coupling. It has been shown that larger correlation leads to larger end to end delay for both interfering routes. Any communication along path $\mathrm{P}$ will not disturb any ongoing communication process at that instant of time can be represented by $\eta(p)=0$. That path is known as zone-disjoint path with respect to all other active path used by other ongoing communications at that instant of time. Otherwise the path is said to be $\eta$ - related path with respect other active paths [11].

\section{B. Network information}

In network each node aware of its neighborhood and communications going on in its neighborhood at that instant of time. With this network status information, each intermediate node adaptively computes maximally zone-disjoint routes (single path or multipath) toward its destination. To track the direction of its neighbors, each node $\mathrm{n}$ periodically collects its neighborhood information and 
store in an angle signal table (AST). $\operatorname{SIGNAL}^{\alpha}{ }_{n, m}(\mathrm{t})$ is the maximum strength of received signal at node $n$ from its neighboring node $\mathrm{m}$ at an angle $\alpha$ at any point of time $t$. The AST of node $n$ will help us to determine the best possible direction of communication with any of its neighbors. Each node $\mathrm{n}$ contains the communication activity status of its neighbors, information of all active nodes in the network, and topology information at that instant of time. Each node broadcasts the information of active nodes, AST of its neighbors, and network topology in the network. Other nodes update their knowledge of network using this broadcast information.

\section{Maximally zone disjoint shortest path routing}

Efficient directional MAC protocol, it lone would not be able to guarantee good system performance unless we have a proper routing strategy in place that exploits the advantages of a directional antenna. Conventional routing schemes generally use a shortest-path routing protocol to improve the network performance in terms of throughput. But the selection of the shortest path for each communication is not at all sufficient to improve network performance under high-traffic load due to coupling effects. To avoid the coupling effect a diverse zone-disjoint path could be selected for a new communication, avoiding the zones already involved in some other communications, then that would definitely improve the throughput by reducing the congestion. Two metrics are used for route selection: correlation factor and propagated hop count. Initially when a packet is transmitted from the source it gives preference to the zone-disjoint path-selection criteria. If a packet already traversed multiple hops, then the progressively shortest hop route toward the destination will be selected. This adaptive route calculation mechanism guarantees the convergence of the proposed routing algorithm

\section{Maximally zone-disjoint multipath routing}

Multipath routing allows to improve the reliability and diminishing the effect of constantly changing topology. It may also help to reduce end-to-end delay and perform load balancing. We have investigated the effect of directional antennas on multipath routing and compared their effectiveness with respect to omni-directional antenna. It has been shown that multipath routing is the best choice when the number of communications is low. However, with an increasing number of communications, single path adaptive routing performs better than multipath adaptive routing. As number of simultaneous communication increases, the number of traffic flow increases, creating more congestion and collision. As a result, the performance of multipath routing degrades as compared to single-path routing, when number of communication is high.

Two nodes $\mathrm{T}$ and $\mathrm{R}$ are communicating using two paths: T-A-B-C-R and T-D-E-F-R as depicted in fig. 3. Because both $A$ and $D$ are within omni-directional transmission range of $\mathrm{T}$ as shown in the figure with a dotted circle, RTS from $\mathrm{T}$ will disable d.

Similarly, because both $\mathrm{C}$ and $\mathrm{F}$ are within omni-directional transmission range of $\mathrm{R}$, CTS from $\mathrm{R}$ will disable both $\mathrm{C}$ and F. So, the lowest possible $\eta$ in the case of omni-directional antennas $\left[\eta_{\min }(\right.$ omni) $]$ with two multipaths between $\mathrm{T}$ and $\mathrm{R}$ is two, if two paths have no common nodes except source and destination. It is not possible to get fully zone-disjoint routes using omni-directional antennas. Hence $\eta_{\min }($ omni $)=2$ and $\eta_{\min }($ dir $)=0$.

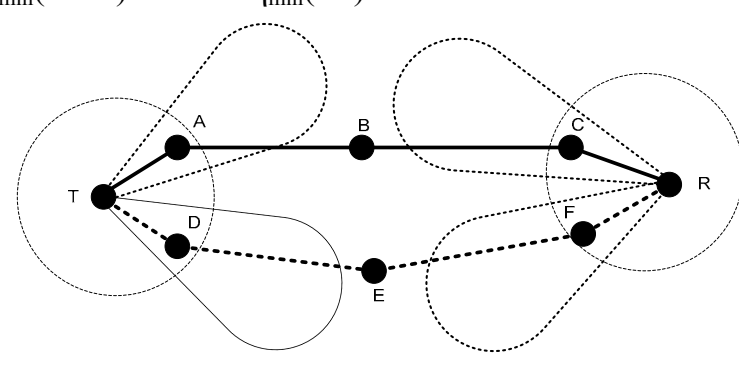

Fig.3: Node disjoint path from source to destination

Even if we get multiple zone-disjoint routes with minimal correlation factor using omni-directional antennas, the best-case packet arrival rate at the destination node will be one packet at every $2 \mathrm{tp}$, where tp is the average delay per hop per packet of a traffic stream on the path $\mathrm{p}$. The best-case assumption is single traffic stream in the network from $\mathrm{T}$ to $\mathrm{R}$ with error-free transmission of packets. Let us refer to Fig. 3 and assume that each node is equipped with an omni-directional antenna and two paths having minimal correlation factor (i.e., $\eta=2$ ) are used for data communication. This implies that nodes $\{\mathrm{A}, \mathrm{B}, \mathrm{C}\}$ and nodes $\{\mathrm{D}, \mathrm{E}, \mathrm{F}\}$ are disjoint. Even if we increase the number of paths between $\mathrm{T}$ and $\mathrm{R}$ beyond two, the situation will not improve with omni-directional antenna.

With directional antenna, it is possible to de-couple these two routes, making them fully zone-disjoint. If each node sets their transmission zone toward its target node only as shown in Fig. 3, then the communication using path T-A-B-C-R will not affect the communication using path T-D-E-F-R. Hence, $\eta$ wherea $\eta_{\min }($ dir $)=0$. If we use directional antenna, the best-case packet arrival rate at destination will be one packet at every tp. When node $\mathrm{A}$ is transmitting a packet to node $\mathrm{B}$, $\mathrm{T}$ can transmit a packet to node $\mathrm{D}$ simultaneously using directional antenna. Thus, destination $\mathrm{R}$ will receive a packet at every time-tick with two zone-disjoint paths using a directional antenna. Two zone-disjoint paths with directional antennas are sufficient to achieve this best-case scenario.

The multipath routing scheme selects a pair of maximally zone-disjoint shortest paths for each communication. Each pair of such path also tries to avoid the nodes that are already handling several other communications. This way multipath routing scheme is capable of balancing the network load, which in turn reduces the possibility of power depletion of some heavily used nodes in the network as well as the probability of congestion. Goal is to distribute the network load along a set of diverse paths to achieve better load balancing through multipath routing for an effective gain in throughput.

\section{PERFormance EVAlUATION}

\section{A. Simulation setup}

We use the Qualnet 5.0 [18] network simulator to simulate the routing protocol using switched beam antenna and ideal 
directional antenna. Qualnet library is a scalable simulation environment for wireless network system using the parallel discrete-event simulation. The simulation has been done in various network scenarios to assess the performance of protocols. The set of parameters used is listed in Table 1. In our simulation model an ad-hoc network of 100 mobile nodes placed randomly within a $1000 \mathrm{mx} 1000 \mathrm{~m}$ area. The coverage area around each node is 250 meters. There were no network partitions throughout the simulation. Each simulation is executed for the 600 seconds of simulation time. Multiple runs with different seed value were conducted for each set of scenario. Fig. 4 show the simulation of 100 nodes with 6 simultaneous communications.

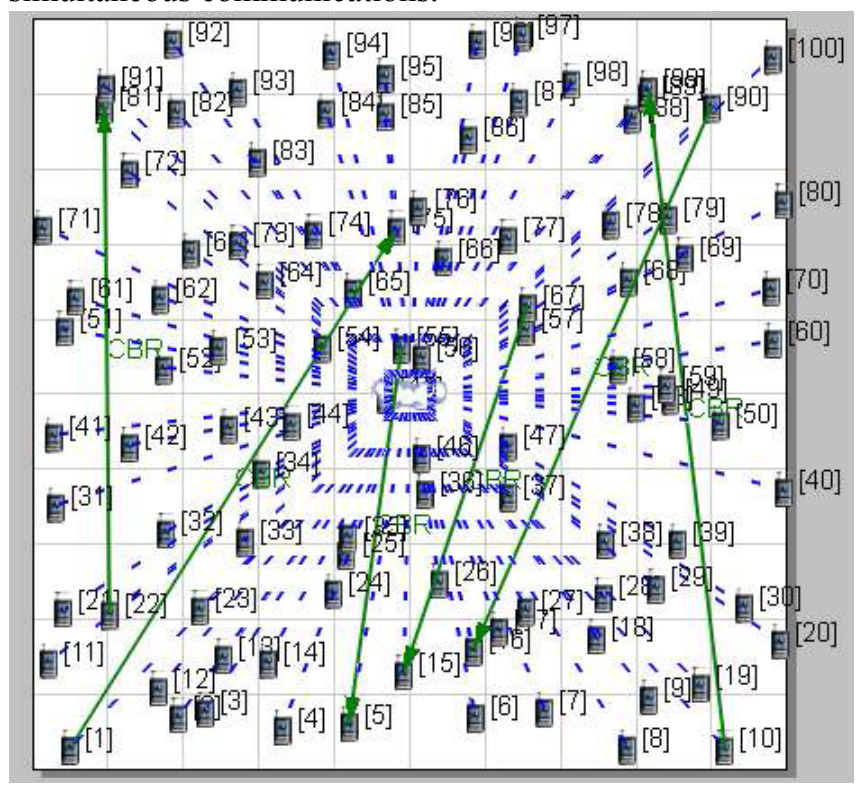

Fig.4: 100 nodes simulation with 6 simultaneous communications in Qualnet.

TABLE I SIMULATION PARAMETERS

\begin{tabular}{ll}
\hline \hline \multicolumn{1}{c}{ Parameters } & \multicolumn{1}{c}{ Values } \\
\hline Area & 1000 X 1000 meters \\
Number of nodes & 100 \\
Transmission power & $10 \mathrm{dBm}$ \\
Receiving threshold & $-81.0 \mathrm{dBm}$ \\
Packet size & $512 \mathrm{bytes}$ \\
CBR packet arrival rate & $2 \mathrm{~ms}$ to $600 \mathrm{~ms}$ \\
Simulation time & $600 \mathrm{sec}$. \\
Transmission range & 250 meters \\
\hline \hline
\end{tabular}

\section{B. Performance with fixed nodes}

We have evaluated the performance of shortest path, single path and multipath routing protocols and compare them with AODV (Adhoc on demand distance vector) that uses the omni-directional antenna. Route coupling has minimum effect when number of simultaneous communications are low. Multi-path outperforms other schemes when the number of communications are low. This is due to fact that, multipath scheme easily finds zone-disjoint paths for multipath traffic flow for a particular communication, when number of simultaneous communications are low. When number of simultaneous communication increases, then due to increase in number of flow it is difficult to find the so many disjoint path. Due to this an increasing number of communication multipath performance degraded as compared to single path. With an increasing number of communications, AODV performance suffers because of low efficiency due to the use of omni-directional antennas and route coupling. Therefore, the throughput of AODV is consistently low as mention in fig. 5 .

The channel utilization can be increased by using the directional antenna in multiple source destination communications. If we select the zone-disjoint path, contention will be reduced and that will balance the network load across all the nodes in the network. If we combine the directional antenna and zone-disjoint route, it will improve the system performance with improved throughput. As shown in fig. 6.

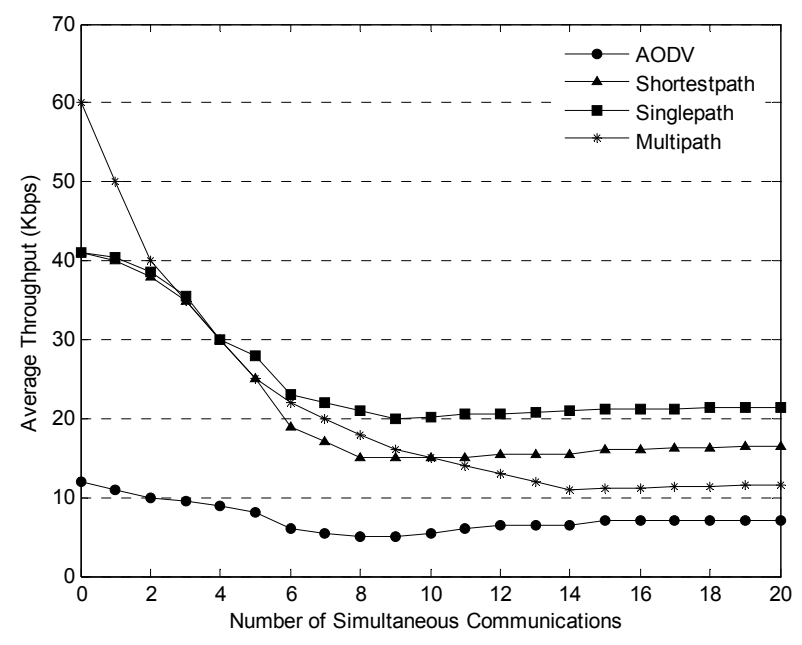

Fig. 5: Average throughput with number of simultaneous communications

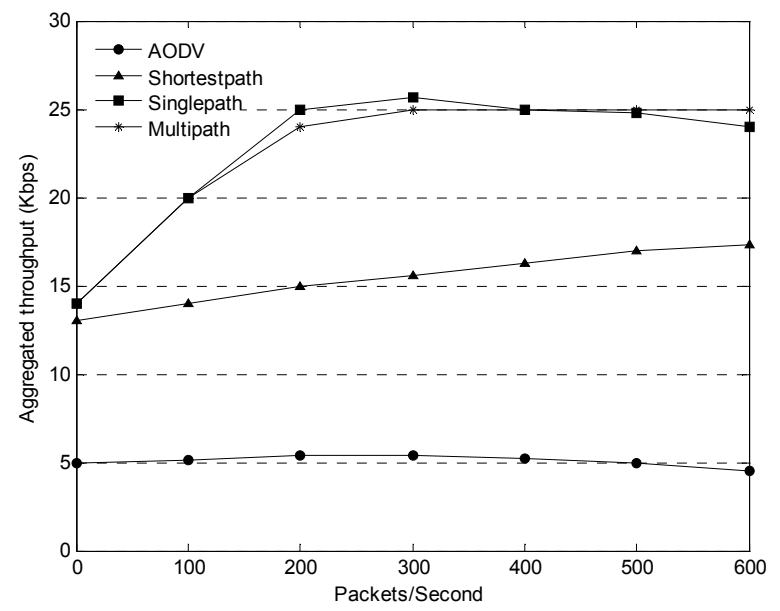

Fig. 6: Average throughput with different packet arrival rate

\section{Performance with mobile nodes}

With mobility, the performance degradation of proposed scheme is much less significant as compare to that of AODV as shown in fig. 7 because this scheme relies on table-driven, adaptive routing, there is no impact of route failure. On the other hand, performance of AODV is heavily dependent on the number of route failures. As mobility increases, route errors due to route failures would increase, degrading the performance of AODV. The only impact of mobility on proposed scheme is that, with increasing mobility, the network status information at any node tends to be less accurate. As a result, a packet may take a longer route to reach its destination because of adaptive route corrections at 
the intermediate nodes. As mentioned earlier, single-path routing performance is little better than multipath, because the number of simultaneous communication.

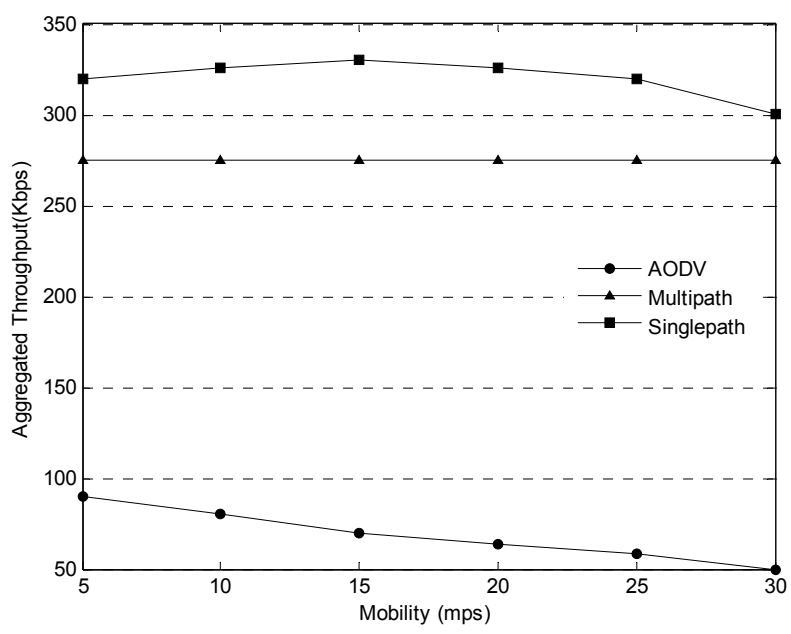

Fig. 7: Aggregated throughput with mobility

\section{CONCLUSION}

Performance of ad hoc networks can be drastically improved by using the directional antennas, with suitable directional MAC and routing protocols. Zone-disjoint routes will reduce route coupling among selected paths and improve throughput. Although increased control overhead in the network, the performance is far better than conventional reactive routing with omni-directional protocols. Multipath routing may not always improve system performance. Multipath routing is the best choice when the number of communication is low. If the number of communication is high, the performance is worse than single-path. Based on this observation, future work will be to investigate the feasibility of hybrid routing where one or two nodes will route data using multipath routing, whereas other nodes will use single-path routing. This may improve the throughput of wireless ad hoc network.

\section{REFERENCES}

[1] Royer, E.M. and Toh, C.K., "A Review of Current Routing Protocols for Ad Hoc Wireless Networks," IEEE Personal Communication, April 1999, p. 46.
[2] Wu, K. and Harms, J., "On-demand Multipath Routing for Mobile Ad Hoc Networks,” EPMCC 2001, Vienna, February 20-22, 2001.

[3] Parkins, C.E. and Royer, E.M., "Ad Hoc On Demand Distance VectorRouting," 2nd IEEE Workshop on Mobile Comp. Sys. and Apps., February 1999, p. 90.

[4] Parkins, C.E. et al., "Ad Hoc On Demand Distance Vector (AODV) Routing,"

http://www.ietf.org/internet-drats/draft-ietf-manet-aodv-03.txt, June 1999. IETF Internet draft.

[5] Pei, G., Gerla, M., and Chen, T.W., "Fisheye State Routing: A Routing Scheme for Ad Hoc Wireless Networks," IEEE International Conference on Communication, New Orleans, Louisiana, June 2000.

[6] Johnson, B. and Maltz, D.A., " Dynamic Source Routing in Ad Hoc Wireless Networks," Mobile Computing, T. Imielinski and H. Korth, Eds., Kluwer, Boston, 1996.

[7] Das, S.R., Perkins, C., and Royer, E., "Performance Comparison of Two On-demand Routing Protocols for Ad Hoc Networks," IEEE INFOCOM 2000, Tel Aviv, March 26-30, 2000.

[8] S. Murthy, J.J. Garcia-Luna-Aceves, "An efficient routing protocol for wireless networks," ACM Mobile Networks and Applications Journal, Special issue on Routing in Mobile Communication Networks, 1996.

[9] Roy Choudhury, R. and Vaidya, N., "Impact of Directional Antennas on Ad Hoc Routing,". 8th Conference on Personal and Wireless Communication $(P W C)$, Venice, September 2003.

[10] Bandyopadhyay, S. et al., "Multipath Routing in Ad Hoc Wireless Networks with Omni Directional and Directional Antenna: A Comparative Study," International Workshop on Distributed Computing, Calcutta, December 27-30, 2003.

[11] Y. Li, H. Man, J. Yu and Y. Yao, "Multipath routing in ad hoc networks using directional antennas,' Advances in Wired and Wireless Communication, 2004 IEEE/Sarnoff Symposium, April 2004, pp $119-122$.

[12] S.J. Lee and M. Gerla, "Split multipath routing with maximally disjoint paths in ad hoc networks," Proceedings of the IEEE ICC, $2001 \mathrm{pp}$. 3201-3205, 2001.

[13] B. Hu and H. Gharavi, "Directional Routing Protocol for. Ad Hoc Networks ,"IET Communication, 2008, Vol. 2, No. 5, pp. 650-657

[14] B. Hu and H. Gharavi, "Directional Antenna for Multipath Ad Hoc Routing," Proceedings of the Fifth IEEE International Symposium on Network Computing and Applications, pp. 27 - 31, 2006

[15] Nasipuri, S. Ye, J. You and R. Hiromoto, "A MAC protocol for mobile ad hoc networks using directional antennas," IEEE Wireless Communications and Networking Conference, Chicago, Illinois, Vol. 3, September 23-28, 2000, pp. 1214-1219.

[16] Z. Huang and C. Shen, "A comparison study of omnidirectional and directional MAC protocols for ad hoc networks," IEEE Globecom'02, 2002

[17] Mineo Takai, Jay Martin, Rajiv Bagrodia and Aifeng Ren, "Directional Virtual Carrier Sensing for Directional Antennas in Mobile Ad Hoc Networks", ACM/SIGMOBILE MobiHoc June 2002.

[18] QualNet Simulator Version 5.0, Scalable Network Technologies, www.scalable-networks.com. 